Proceedings of the Edinburgh Mathematical Society (2003) 46, 45-61 (C)

DOI:10.1017/S001309150200010X Printed in the United Kingdom

\title{
SEPARATING MILLIKEN-TAYLOR SYSTEMS WITH NEGATIVE ENTRIES
}

\author{
NEIL HINDMAN ${ }^{1}$, IMRE LEADER ${ }^{2}$ AND DONA STRAUSS ${ }^{3}$ \\ ${ }^{1}$ Department of Mathematics, Howard University, \\ Washington, DC 20059, USA (nhindman@aol.com) \\ ${ }^{2}$ Department of Pure Mathematics and Mathematical Statistics, \\ Centre for Mathematical Sciences, University of Cambridge, \\ Wilberforce Road, Cambridge CB3 OWB, UK \\ (i.leader@dpmms.cam.ac.uk) \\ ${ }^{3}$ Department of Pure Mathematics, University of Hull, \\ Hull HU6 7RX, UK (d.strauss@maths.hull.ac.uk)
}

(Received 25 January 2002)

Dedicated to to the memory of Walter Deuber.

Abstract Given a finite sequence $\boldsymbol{a}=\left\langle a_{i}\right\rangle_{i=1}^{n}$ in $\mathbb{N}$ and a sequence $\left\langle x_{t}\right\rangle_{t=1}^{\infty}$ in $\mathbb{N}$, the Milliken-Taylor system generated by $\boldsymbol{a}$ and $\left\langle x_{t}\right\rangle_{t=1}^{\infty}$ is

$$
\begin{aligned}
\operatorname{MT}\left(\boldsymbol{a},\left\langle x_{t}\right\rangle_{t=1}^{\infty}\right)=\left\{\sum_{i=1}^{n} a_{i} \cdot \sum_{t \in F_{i}} x_{t}: F_{1}, F_{2}, \ldots, F_{n}\right. \text { are finite non-empty } \\
\text { subsets of } \left.\mathbb{N} \text { with } \max F_{i}<\min F_{i+1} \text { for } i<n\right\} .
\end{aligned}
$$

It is known that Milliken-Taylor systems are partition regular but not consistent. More precisely, if $\boldsymbol{a}$ and $\boldsymbol{b}$ are finite sequences in $\mathbb{N}$, then, except in trivial cases, there is a partition of $\mathbb{N}$ into two cells, neither of which contains $\operatorname{MT}\left(\boldsymbol{a},\left\langle x_{t}\right\rangle_{t=1}^{\infty}\right) \cup \operatorname{MT}\left(\boldsymbol{b},\left\langle y_{t}\right\rangle_{t=1}^{\infty}\right)$ for any sequences $\left\langle x_{t}\right\rangle_{t=1}^{\infty}$ and $\left\langle y_{t}\right\rangle_{t=1}^{\infty}$.

Our aim in this paper is to extend the above result to allow negative entries in $\boldsymbol{a}$ and $\boldsymbol{b}$. We do so with a proof which is significantly shorter and simpler than the original proof which applied only to positive coefficients. We also derive some results concerning the existence of solutions of certain linear equations in $\beta \mathbb{Z}$. In particular, we show that the ability to guarantee the existence of $\operatorname{MT}\left(\boldsymbol{a},\left\langle x_{t}\right\rangle_{t=1}^{\infty}\right) \cup \operatorname{MT}\left(\boldsymbol{b},\left\langle y_{t}\right\rangle_{t=1}^{\infty}\right)$ in one cell of a partition is equivalent to the ability to find idempotents $p$ and $q$ in $\beta \mathbb{N}$ such that $a_{1} \cdot p+a_{2} \cdot p+\cdots+a_{n} \cdot p=b_{1} \cdot q+b_{2} \cdot q+\cdots+b_{m} \cdot q$, and thus determine exactly when the latter has a solution.

Keywords: Stone-Čech compactification; image-partition regularity; Milliken-Taylor systems

AMS 2000 Mathematics subject classification: Primary 05D10

Secondary 22A $15 ; 54 \mathrm{H} 13$

\section{Introduction}

There are striking differences between finite and infinite partition-regular systems of linear expressions. To make this assertion precise, we remind the reader of the notion of an image-partition-regular matrix. (We are taking $\mathbb{N}$ to be the set of positive integers.) 
Definition 1.1. Let $A$ be a (finite or infinite) matrix with entries from $\mathbb{Z}$ and only finitely many non-zero entries on each row. Then $A$ is image-partition regular if and only if whenever $\mathbb{Z}$ is partitioned into finitely many classes (or is finitely coloured), there exists a vector $\boldsymbol{x}$ of the appropriate size with entries from $\mathbb{N}$ such that all entries of $A \boldsymbol{x}$ are in the same class (or are monochrome).

Image-partition-regular matrices arise naturally in Ramsey Theory. For example, van der Waerden's Theorem and Schur's Theorem are naturally stated as the assertion that certain matrices are image-partition regular. See [4], [7] or [5, Chapter 15] for more extensive discussions of image-partition-regular matrices. (One of the major differences between finite and infinite image-partition-regular matrices is that the former have been completely characterized [4], while the characterization of infinite image-partition-regular matrices is a vexing open problem. However, we are not concerned with this difference in this paper.)

It is a consequence of a result of Deuber [2] and some results from [4] that whenever $A$ and $B$ are finite image-partition-regular matrices, then so is the matrix

$$
\left(\begin{array}{ll}
A & \mathbf{0} \\
\mathbf{0} & B
\end{array}\right) \text {. }
$$

That is, whenever $\mathbb{Z}$ is finitely coloured, there must exist vectors $\boldsymbol{x}$ and $\boldsymbol{y}$ of the appropriate size with entries from $\mathbb{N}$ such that all entries of $A \boldsymbol{x}$ and $B \boldsymbol{y}$ have the same colour. This is far from the case with infinite image-partition-regular matrices. To further this discussion, we introduce the notion of Milliken-Taylor systems. Given a set $A$, we denote the set of finite non-empty subsets of $A$ by $\mathcal{P}_{f}(A)$.

Definition 1.2. Let $\boldsymbol{a}=\left\langle a_{i}\right\rangle_{i=1}^{n}$ be a finite sequence in $\mathbb{Z} \backslash\{0\}$ and let $\left\langle x_{t}\right\rangle_{t=1}^{\infty}$ be a sequence in $\mathbb{N}$. The Milliken-Taylor system $\operatorname{MT}\left(\boldsymbol{a},\left\langle x_{t}\right\rangle_{t=1}^{\infty}\right)$ generated by $\boldsymbol{a}$ and $\left\langle x_{t}\right\rangle_{t=1}^{\infty}$ is

$$
\left\{\sum_{i=1}^{n} a_{i} \cdot \sum_{t \in F_{i}} x_{t}: F_{1}, F_{2}, \ldots, F_{n} \in \mathcal{P}_{f}(\mathbb{N}) \text { and } \max F_{i}<\min F_{i+1} \text { for } i<n\right\} .
$$

Milliken-Taylor systems are so named because their partition regularity follows immediately from the Milliken-Taylor Theorem (see [9, Theorem 2.2] and [10, Lemma 2.2]).

Definition 1.3. Let $\left\langle y_{n}\right\rangle_{n=1}^{\infty}$ and $\left\langle x_{n}\right\rangle_{n=1}^{\infty}$ be sequences in $\mathbb{N}$. The sequence $\left\langle x_{n}\right\rangle_{n=1}^{\infty}$ is a sum subsystem of $\left\langle y_{n}\right\rangle_{n=1}^{\infty}$ if and only if there is a sequence $\left\langle H_{n}\right\rangle_{n=1}^{\infty}$ in $\mathcal{P}_{f}(\mathbb{N})$ with $\max H_{n}<\min H_{n+1}$ for each $n \in \mathbb{N}$ and $x_{n}=\sum_{\ell \in H_{n}} y_{\ell}$ for each $n \in \mathbb{N}$.

Notice that if $\left\langle x_{n}\right\rangle_{n=1}^{\infty}$ is a sum subsystem of $\left\langle y_{n}\right\rangle_{n=1}^{\infty}$, then

$$
\operatorname{FS}\left(\left\langle x_{n}\right\rangle_{n=1}^{\infty}\right) \subseteq \operatorname{FS}\left(\left\langle y_{n}\right\rangle_{n=1}^{\infty}\right)
$$

where

$$
\operatorname{FS}\left(\left\langle x_{n}\right\rangle_{n=1}^{\infty}\right)=\left\{\sum_{n \in F} x_{n}: F \in \mathcal{P}_{f}(\mathbb{N})\right\}=\operatorname{MT}\left(\langle 1\rangle,\left\langle x_{n}\right\rangle_{n=1}^{\infty}\right)
$$


Not only are Milliken-Taylor systems partition regular, but in fact the following stronger result is true.

Theorem 1.4. Let $\boldsymbol{a}$ be a finite sequence in $\mathbb{N}$ and let $\left\langle y_{n}\right\rangle_{n=1}^{\infty}$ be a sequence in $\mathbb{N}$. Let $r \in \mathbb{N}$ and let $\mathbb{N}=\bigcup_{i=1}^{r} B_{i}$. Then there exist $i \in\{1,2, \ldots, r\}$ and a sum subsystem $\left\langle x_{n}\right\rangle_{n=1}^{\infty}$ of $\left\langle y_{n}\right\rangle_{n=1}^{\infty}$ with $\operatorname{MT}\left(\boldsymbol{a},\left\langle x_{n}\right\rangle_{n=1}^{\infty}\right) \subseteq B_{i}$.

Proof. See [3, Theorem 2.5].

We can now describe the striking difference between finite and infinite image-partitionregular matrices, with which we are concerned. Consider, for example, the matrix $A$ whose rows consist of all rows with entries from $\{0,1,2\}$ with only finitely many nonzero entries, at least one 1 , at least one 2 , and all occurrences of 1 before any occurrences of 2 . Consider also the matrix $B$ whose rows consist of all rows with entries from $\{0,1,2\}$ with only finitely many non-zero entries, at least one 1 , at least one 2 , and all occurrences of 2 before any occurrences of 1 . Then, given a sequence $\boldsymbol{x}=\left\langle x_{n}\right\rangle_{n=1}^{\infty}$, the set of entries of $A \boldsymbol{x}$ is $\operatorname{MT}\left(\langle 1,2\rangle,\left\langle x_{n}\right\rangle_{n=1}^{\infty}\right)$ and the set of entries of $B \boldsymbol{x}$ is $\operatorname{MT}\left(\langle 2,1\rangle,\left\langle x_{n}\right\rangle_{n=1}^{\infty}\right)$. Thus, by Theorem 1.4, the matrices $A$ and $B$ are image-partition regular. On the other hand, it was shown in $[\mathbf{3}$, Theorem 3.3] that

$$
\left(\begin{array}{ll}
A & \mathbf{0} \\
\mathbf{0} & B
\end{array}\right)
$$

is not image-partition regular. And we can say more. We know exactly when such matrices can be combined to yield an image-partition-regular matrix.

Definition 1.5. Let $\boldsymbol{a}=\left\langle a_{i}\right\rangle_{i=1}^{n}$ be a finite sequence. Then $\boldsymbol{a}$ is a compressed sequence if and only if $\boldsymbol{a}$ has no adjacent repeated terms.

We note that, as far as partition regularity is concerned, we lose no generality by restricting our attention to compressed sequences $\boldsymbol{a}$. In the following lemma, if we had $\boldsymbol{a}=\langle 2,-3,-3,1,1,1,1,2\rangle$, then we would have $\boldsymbol{c}=\langle 2,-3,1,2\rangle$.

Lemma 1.6. Let $\boldsymbol{a}$ be a finite sequence in $\mathbb{Z} \backslash\{0\}$ and let $\boldsymbol{c}$ be the compressed sequence obtained by deleting adjacent repetitions of terms. Let $\left\langle y_{n}\right\rangle_{n=1}^{\infty}$ be a sequence in $\mathbb{N}$. Then there is a sum subsystem $\left\langle x_{n}\right\rangle_{n=1}^{\infty}$ of $\left\langle y_{n}\right\rangle_{n=1}^{\infty}$ such that $\operatorname{MT}\left(\boldsymbol{c},\left\langle x_{n}\right\rangle_{n=1}^{\infty}\right) \subseteq$ $\operatorname{MT}\left(\boldsymbol{a},\left\langle y_{n}\right\rangle_{n=1}^{\infty}\right)$.

Proof. Let $m$ be the length of $\boldsymbol{a}$ and for $k \in \mathbb{N}$, let $H_{k}=\{(k-1) m+1,(k-1) m+$ $2, \ldots, k m\}$ and let $x_{k}=\sum_{t \in H_{k}} y_{t}$.

The main result of $[\mathbf{3}]$ determined precisely when one could guarantee Milliken-Taylor systems for $\boldsymbol{a}$ and $\boldsymbol{b}$ in the same cell of an arbitrary partition of $\mathbb{N}$, provided that the entries of $\boldsymbol{a}$ and $\boldsymbol{b}$ are positive. 
Theorem 1.7. Let $\boldsymbol{a}$ and $\boldsymbol{b}$ be finite compressed sequences with entries from $\mathbb{N}$. The following statements are equivalent.

(a) Whenever $r \in \mathbb{N}$ and $\mathbb{N}=\bigcup_{i=1}^{r} B_{i}$, there exist $i \in\{1,2, \ldots, r\}$ and sequences $\left\langle x_{n}\right\rangle_{n=1}^{\infty}$ and $\left\langle y_{n}\right\rangle_{n=1}^{\infty}$ with $\operatorname{MT}\left(\boldsymbol{a},\left\langle x_{n}\right\rangle_{n=1}^{\infty}\right) \cup \operatorname{MT}\left(\boldsymbol{b},\left\langle y_{n}\right\rangle_{n=1}^{\infty}\right) \subseteq B_{i}$.

(b) There is a positive rational number $\alpha$ such that $\boldsymbol{b}=\alpha \cdot \boldsymbol{a}$.

Proof. See [3, Theorems 3.2 and 3.3].

In the definition of partition regularity of matrices, the requirement that the entries of $\left\langle x_{n}\right\rangle_{n=1}^{\infty}$ be positive is there because that is desired in the typical classical Ramseytheoretic applications. In [3], Deuber et al. did not ask what happens when the entries of $\boldsymbol{a}$ are allowed to be negative. Had they asked this question, they could have presented the following result, which was first stated in [7, Corollary 3.6].

Theorem 1.8. Let $\boldsymbol{a}$ be a finite sequence in $\mathbb{Z} \backslash\{0\}$ and let $\left\langle y_{n}\right\rangle_{n=1}^{\infty}$ be a sequence in $\mathbb{N}$. Let $r \in \mathbb{N}$ and let $\mathbb{Z}=\bigcup_{i=1}^{r} B_{i}$. Then there exist $i \in\{1,2, \ldots, r\}$ and a sum subsystem $\left\langle x_{n}\right\rangle_{n=1}^{\infty}$ of $\left\langle y_{n}\right\rangle_{n=1}^{\infty}$ with $\operatorname{MT}\left(\boldsymbol{a},\left\langle x_{n}\right\rangle_{n=1}^{\infty}\right) \subseteq B_{i}$.

Proof. The proof of [3, Theorem 2.5] may be copied verbatim.

Furthermore, if in Theorem 1.7 the entries of $\boldsymbol{a}$ and $\boldsymbol{b}$ are allowed to be negative, then one may take the proof that (b) implies (a) directly from the proof of [3, Theorem 3.2].

The matter of the proof that (a) implies (b) in the revised Theorem 1.7 is considerably more complicated. In the first place, the proof of [3, Theorem 3.3] is lengthy and at least moderately intricate. In the second place, that proof does not easily accommodate the inclusion of negative numbers. The reason has to do with the difference between the addition and subtraction algorithms in our ordinary arithmetic (to a specified positive base).

It is easy to see that, given $p \in \mathbb{N}$ and a sequence $\left\langle y_{n}\right\rangle_{n=1}^{\infty}$ in $\mathbb{N}$, there is a sum subsystem $\left\langle x_{n}\right\rangle_{n=1}^{\infty}$ of $\left\langle y_{n}\right\rangle_{n=1}^{\infty}$ with the property that for any $t, n \in \mathbb{N}$, if $x_{n} \leqslant p^{t}$, then $p^{t+1}$ divides $x_{n+1}$, and consequently there is no carrying when $x_{n}$ and $x_{n+1}$ are added in base $p$ arithmetic. This fact allowed a colouring of $\mathbb{N}$ based on patterns which occurred in the base $p$ expansion of members of $\mathbb{N}$ which could separate $\operatorname{MT}\left(\boldsymbol{a},\left\langle x_{n}\right\rangle_{n=1}^{\infty}\right)$ from $\operatorname{MT}\left(\boldsymbol{b},\left\langle y_{n}\right\rangle_{n=1}^{\infty}\right)$ for any sequences $\left\langle x_{n}\right\rangle_{n=1}^{\infty}$ and $\left\langle y_{n}\right\rangle_{n=1}^{\infty}$, as long as one did not have $\boldsymbol{b}=\alpha \cdot \boldsymbol{a}$ for any positive rational $\alpha$.

However, even under these conditions, there is borrowing when $x_{n}$ is subtracted from $x_{n+1}$. The fact that the string of zeros between the least significant digit of $x_{n+1}$ and the most significant digit of $x_{n}$ is replaced by a string of $(p-1)$ s is not a serious problem, but the change in the least significant digit of $x_{n+1}$ caused by the borrowing seriously disrupts the patterns of digits. This fact caused us significant problems. Then we recalled a lecture that two of us heard at the University of Sheffield in 1996 at which Behzad Bordbar discussed some joint research with John Pym [1] which used the fact that any integer (positive, zero or negative) has a unique expansion to the base -2 (using only the digits 0 and 1). A moment's reflection will convince the reader that the same statement 
is true with regard to base $-p$, using the digits $\{0,1, \ldots, p-1\}$. There are two important properties of this expansion. The first is that a number is divisible by $p^{t}$ if and only if the rightmost $t$ digits are 0 . The second is that, when $t \in \mathbb{N}, x, y \in \mathbb{Z},|x| \leqslant p^{t}$ and $p^{t+1}$ divides $y$, then there is no carrying and no borrowing when $x$ and $y$ are added in base $-p$. This fact allows us to modify the construction of $[\mathbf{3}]$ and establish the analogue of Theorem 1.7 which allows entries of $\boldsymbol{a}$ and $\boldsymbol{b}$ to be negative.

In $\S 2$ of this paper we present some relevant facts about negative base arithmetic and some special functions that we will use. In $\S 3$ we complete the proof of the analogue of Theorem 1.7. In $\S 4$ we present additional equivalent conditions dealing with the solution of certain linear equations in the Stone-Cech compactification of $\mathbb{Z}$.

\section{Arithmetic in base $-p$}

We begin with the description of the base $-p$ expansion and some routine facts about that expansion, whose proofs we omit. (We take $\omega=\mathbb{N} \cup\{0\}$.)

Lemma 2.1. Let $p \in \mathbb{N}$ with $p \geqslant 2$. For every $x \in \mathbb{Z}$, there exists a unique function $\gamma_{p, x}: \omega \rightarrow\{0,1, \ldots, p-1\}$ (with $\left\{t \in \omega: \gamma_{p, x}(t) \neq 0\right\}$ finite) such that

$$
x=\sum_{t=0}^{\infty} \gamma_{p, x}(t) \cdot(-p)^{t} .
$$

If $x>0$, then $\max \left\{t \in \omega: \gamma_{p, x}(t) \neq 0\right\}$ is even, and if $x<0$, then $\max \{t \in \omega:$ $\left.\gamma_{p, x}(t) \neq 0\right\}$ is odd. For any $x \in \mathbb{Z} \backslash\{0\}$ and any $n \in \mathbb{N}, p^{n}$ divides $x$ if and only if $\min \left\{t \in \omega: \gamma_{p, x}(t) \neq 0\right\} \geqslant n$.

Given $x \in \mathbb{Z} \backslash\{0\}$ and $p \in \mathbb{N} \backslash\{1\}$ if $\alpha=\max \left\{t \in \omega: \gamma_{p, x}(t) \neq 0\right\}$, we refer to $\gamma_{p, x}(\alpha)$ as the most significant digit of $x$ in the base $-p$ expansion and we refer to $\alpha$ as the location of the most significant digit. Similarly, if $\delta=\min \left\{t \in \omega: \gamma_{p, x}(t) \neq 0\right\}$, then $\gamma_{p, x}(\delta)$ is the least significant digit and $\delta$ is its location.

Lemma 2.2. Let $p \in \mathbb{N} \backslash\{1\}$, let $t \in \mathbb{N}$, and let $x \in \mathbb{Z} \backslash\{0\}$. If $x$ is expressible in base $-p$ with the most significant digit in location $t$, then

$$
\frac{p^{t}}{p+1}<|x|<\frac{p^{t+2}}{p+1}
$$

Proof. If $t$ is even, this follows easily from the inequalities:

$$
p^{t}-(p-1)\left(p^{t-1}+p^{t-3}+\cdots+p\right) \leqslant x \leqslant(p-1)\left(p^{t}+p^{t-2}+\cdots+p^{2}+1\right) .
$$

If $t$ is odd, our claim then follows from the inequalities:

$$
\frac{p^{t+1}}{p+1}<-p x=p|x|<\frac{p^{t+3}}{p+1}
$$


Corollary 2.3. Let $a, x \in \mathbb{Z} \backslash\{0\}$, with $|a|<p$. If the most significant digits of $x$ and $a x$ in their base $-p$ expansions occur in positions $t$ and $u$, respectively, then $t-1 \leqslant u \leqslant t+2$.

Proof. This is immediate from the inequalities:

$$
\frac{p^{t}}{p+1}<|x| \leqslant|a x|<\frac{p^{t+3}}{p+1}
$$

and

$$
\frac{p^{u}}{p+1}<|a x|<\frac{p^{u+2}}{p+1}
$$

We now introduce some special functions which we will use to define colourings of $\mathbb{Z}$.

Definition 2.4. Let $p \in \mathbb{N} \backslash\{1\}$.

(a) For each $x \in \mathbb{Z} \backslash\{0\}$, we define $\rho_{p}(x) \in\{1,2, \ldots, p-1\}$ to be the least significant digit in the base $-p$ expansion of $x$.

(b) If $x \in \mathbb{Z}$ with $|x|>p^{11}$, we define $\lambda_{p}(x) \in\{0,1,2, \ldots, p-1\}^{11}$ by $\lambda_{p}(x)=$ $\left(v_{1}, v_{2}, \ldots, v_{11}\right)$, where $v_{1} v_{2} \cdots v_{11}$ occurs in the base $-p$ expansion of $x$ with $v_{1}$ at a location $t$ which is a multiple of 6 , and the most significant digit of the expansion occurs at location $s$ with $t-5 \leqslant s \leqslant t$.

Notice that if $\lambda_{p}(x)=\lambda_{p}(y)$, then the most significant digits of $x$ and $y$ occur in positions that are congruent $\bmod 6($ hence $\bmod 2)$ and thus $x$ and $y$ have the same sign.

Lemma 2.5. Let $p \geqslant 3$ be a prime. Let $x, y \in \mathbb{Z} \backslash\{0\}$ and let $a, b, c \in \mathbb{Z} \backslash\{0\}$ satisfy $|a|,|b|,|c|,|a-b|<p$.

(i) If $\rho_{p}(a x)=\rho_{p}(b x)$, then $a=b$.

(ii) If $|x|,|y|>p^{11}$ and if $\lambda_{p}(c x)=\lambda_{p}(c y)$ and $\lambda_{p}(a x)=\lambda_{p}(b y)$, then $a=b$.

Proof. (i) If $\rho_{p}(x)=\rho_{p}(y)=u$, then $a u \equiv b u(\bmod p)$ and so $a=b$.

(ii) Let $t, t^{\prime}, u, u^{\prime}, v, v^{\prime}$ denote the locations of the most significant digits of $x, y, a x$, $b y, c x, c y$, respectively, in their base $-p$ expansions.

We may suppose that $u=u^{\prime}$. If $u^{\prime}>u$, we can replace $x$ by $(-p)^{u^{\prime}-u} x$. Since $u \equiv u^{\prime}$ $(\bmod 6)$, this does not alter $\lambda_{p}(a x)$ or $\lambda_{p}(c x)$. If $u^{\prime}<u$, we can replace $y$ by $(-p)^{u-u^{\prime}} y$.

We claim that $v=v^{\prime}$. We suppose that $t^{\prime} \geqslant t$, the other case being similar. By Corollary $2.3, t^{\prime}-1 \leqslant u^{\prime}=u \leqslant t+2$. So $t^{\prime} \leqslant t+3$. However, $x$ and $y$ have the same sign, because $c x$ and $c y$ have the same sign, and therefore $t$ and $t^{\prime}$ have the same parity. Thus $t^{\prime} \leqslant t+2$. Now $t-1 \leqslant v \leqslant t+2$ and $t-1 \leqslant t^{\prime}-1 \leqslant v^{\prime} \leqslant t^{\prime}+2 \leqslant t+4$. Since $v \equiv v^{\prime}(\bmod 6), v=v^{\prime}$. 
We have

$$
a x=w_{1}(-p)^{u}+w_{2}(-p)^{u-1}+\cdots+w_{6}(-p)^{u-5}+z
$$

and

$$
b y=w_{1}(-p)^{u}+w_{2}(-p)^{u-1}+\cdots+w_{6}(-p)^{u-5}+z^{\prime},
$$

where $w_{1}, w_{2}, w_{3}, w_{4}, w_{5}, w_{6} \in\{0,1,2, \ldots, p-1\}$ and

$$
|z|,\left|z^{\prime}\right|<\frac{p^{u-4}}{p+1} \leqslant \frac{p^{t-2}}{p+1} .
$$

So $|a x-b y|<2\left(p^{t-2} /(p+1)\right)$. Similarly, $|x-y| \leqslant|c(x-y)|<2\left(p^{t-2} /(p+1)\right)$ and so $|b x-b y|<2\left(p^{t-1} /(p+1)\right)$. Thus

$$
|(b-a) x| \leqslant 2 \frac{p^{t-1}}{p+1}+2 \frac{p^{t-2}}{p+1}<\frac{p^{t}}{p+1}<|x|
$$

so $b=a$.

We remark that it is the above proof which forces us to require 11 digits in $\lambda_{p}(x)$. If $\lambda_{p}(a x)=\lambda_{p}(b x)=\left(v_{1}, v_{2}, \ldots, v_{11}\right)$, then one could have $u=v_{6}$, in which case $\left(w_{1}, w_{2}, \ldots, w_{6}\right)=\left(v_{6}, v_{7}, \ldots, v_{11}\right)$.

\section{Separating $\operatorname{MT}\left(a,\left\langle x_{n}\right\rangle_{n=1}^{\infty}\right)$ from $\operatorname{MT}\left(b,\left\langle y_{n}\right\rangle_{n=1}^{\infty}\right)$}

We shall be concerned in this section with establishing the generalization of Theorem 1.7 which allows entries of $\boldsymbol{a}$ and $\boldsymbol{b}$ to be negative. The proof that we present of the generalization turns out to be significantly simpler and shorter than the original proof.

Theorem 3.1. Let $\boldsymbol{a}$ and $\boldsymbol{b}$ be finite compressed sequences with entries from $\mathbb{Z} \backslash\{0\}$. The following statements are equivalent.

(a) Whenever $r \in \mathbb{N}$ and $\mathbb{Z}=\bigcup_{i=1}^{r} B_{i}$, there exist $i \in\{1,2, \ldots, r\}$ and sequences $\left\langle x_{t}\right\rangle_{t=1}^{\infty}$ and $\left\langle y_{t}\right\rangle_{t=1}^{\infty}$ in $\mathbb{N}$ with $\operatorname{MT}\left(\boldsymbol{a},\left\langle x_{t}\right\rangle_{t=1}^{\infty}\right) \cup \operatorname{MT}\left(\boldsymbol{b},\left\langle y_{t}\right\rangle_{t=1}^{\infty}\right) \subseteq B_{i}$.

(b) There is a positive rational number $\alpha$ such that $\boldsymbol{b}=\alpha \cdot \boldsymbol{a}$.

Proof. (b) implies (a). Pick positive integers $m$ and $n$ such that $\alpha=m / n$ and let $\boldsymbol{d}=m \boldsymbol{a}$. Assume that $r \in \mathbb{N}$ and $\mathbb{Z}=\bigcup_{i=1}^{r} B_{i}$. By Theorem 1.8, pick an $i \in\{1,2, \ldots, r\}$ and a sequence $\left\langle z_{t}\right\rangle_{t=1}^{\infty}$ in $\mathbb{N}$ such that $\operatorname{MT}\left(\boldsymbol{d},\left\langle z_{t}\right\rangle_{t=1}^{\infty}\right) \subseteq B_{i}$. For each $t \in \mathbb{N}$, let $x_{t}=m z_{t}$ and let $y_{t}=n z_{t}$. Then $\operatorname{MT}\left(\boldsymbol{a},\left\langle x_{t}\right\rangle_{t=1}^{\infty}\right)=\operatorname{MT}\left(\boldsymbol{b},\left\langle y_{t}\right\rangle_{t=1}^{\infty}\right)=\operatorname{MT}\left(\boldsymbol{d},\left\langle z_{t}\right\rangle_{t=1}^{\infty}\right)$.

The proof that (a) implies (b) will include several definitions and lemmas. We assume that we have compressed sequences $\boldsymbol{a}=\left\langle a_{1}, a_{2}, \ldots, a_{n}\right\rangle$ and $\boldsymbol{b}=\left\langle b_{1}, b_{2}, \ldots, b_{m}\right\rangle$ with entries from $\mathbb{Z} \backslash\{0\}$ such that whenever $\mathbb{Z}$ is finitely coloured there exist sequences $\left\langle x_{t}\right\rangle_{t=1}^{\infty}$ and $\left\langle y_{t}\right\rangle_{t=1}^{\infty}$ in $\mathbb{N}$ with $\operatorname{MT}\left(\boldsymbol{a},\left\langle x_{t}\right\rangle_{t=1}^{\infty}\right) \cup \operatorname{MT}\left(\boldsymbol{b},\left\langle y_{t}\right\rangle_{t=1}^{\infty}\right)$ monochrome. 
We choose a prime number $p$ such that $p>2\left|a_{i}\right|+2\left|b_{j}\right|$ for every $i \in\{1,2, \ldots, n\}$ and every $j \in\{1,2, \ldots, m\}$. We also choose an even positive integer $k$ such that $k>$ $2 m+2 n$. We use $\pi: \mathbb{Z} \rightarrow \mathbb{Z}_{k}$ for the canonical homomorphism, and we represent $\mathbb{Z}_{k}$ as $\{0,1, \ldots, k-1\}$.

Definition 3.2. Let $x \in \mathbb{N}$. Then $\operatorname{supp}(x)=\left\{t \in \omega: \gamma_{p, x}(t) \neq 0\right\}$.

In the above definition we suppress the dependence of $\operatorname{supp}(x)$ on $p$, because $p$ will remain fixed throughout the remainder of this section. Similarly, we shall write $\rho(x)$ and $\lambda(x)$ instead of $\rho_{p}(x)$ and $\lambda_{p}(x)$

Lemma 3.3. Let $\left\langle x_{t}\right\rangle_{t=1}^{\infty}$ be an arbitrary sequence in $\mathbb{N}$.

(a) Given any $b \in \mathbb{N}$, there is a sum subsystem $\left\langle u_{t}\right\rangle_{t=1}^{\infty}$ of $\left\langle x_{t}\right\rangle_{t=1}^{\infty}$ such that $\operatorname{FS}\left(\left\langle u_{t}\right\rangle_{t=1}^{\infty}\right) \subseteq b \mathbb{N}$.

(b) There is a sum subsystem $\left\langle y_{t}\right\rangle_{t=1}^{\infty}$ of $\left\langle x_{t}\right\rangle_{t=1}^{\infty}$ such that for each $t \in \mathbb{N}$,

$$
\min \left(\operatorname{supp}\left(y_{t+1}\right)\right) \geqslant 13+\max \left(\operatorname{supp}\left(y_{t}\right)\right) .
$$

(c) Given any finite colouring of $\mathbb{N}$ and any $b \in \mathbb{N}$, there is a sum subsystem $\left\langle z_{t}\right\rangle_{t=1}^{\infty}$ of $\left\langle x_{t}\right\rangle_{t=1}^{\infty}$ such that $\mathrm{FS}\left(\left\langle z_{t}\right\rangle_{t=1}^{\infty}\right) \subseteq b \mathbb{N}, \mathrm{FS}\left(\left\langle z_{t}\right\rangle_{t=1}^{\infty}\right)$ is monochrome, and for each $t \in \mathbb{N}, \min \left(\operatorname{supp}\left(z_{t+1}\right)\right) \geqslant 13+\max \left(\operatorname{supp}\left(y_{t}\right)\right)$.

Proof. (a) By thinning, we may presume that $x_{t} \equiv x_{s}(\bmod b)$ for all $t, s \in \mathbb{N}$. For each $s \in \mathbb{N}$, let $H_{s}=\{s b, s b+1, s b+2, \ldots,(s+1) b-1\}$ and let $u_{s}=\sum_{t \in H_{s}} x_{t}$.

(b) Let $H_{1}=\{1\}$ and let $y_{1}=x_{1}$. Inductively, given $s \in \mathbb{N}$, assume that we have chosen $H_{s}$ and $y_{s}=\sum_{t \in H_{s}} x_{t}$. Let $r=13+\max \left(\operatorname{supp}\left(y_{s}\right)\right)$. Choose $H_{s+1} \subseteq\{i \in \mathbb{N}$ : $\left.i>\max \left(H_{s}\right)\right\}$ such that $\left|H_{s+1}\right|=p^{r}$ and $x_{i} \equiv x_{j}\left(\bmod p^{r}\right)$ for all $i, j \in H_{s+1}$. Let $y_{s+1}=\sum_{t \in H_{s+1}} x_{t}$. Then $p^{r}$ divides $y_{s+1}$, so $\min \left(\operatorname{supp}\left(y_{s+1}\right)\right) \geqslant r$.

(c) Using (a), choose a sum subsystem $\left\langle u_{t}\right\rangle_{t=1}^{\infty}$ of $\left\langle x_{t}\right\rangle_{t=1}^{\infty}$ such that $\operatorname{FS}\left(\left\langle u_{t}\right\rangle_{t=1}^{\infty}\right) \subseteq$ $b \mathbb{N}$. Using (b), choose a sum subsystem $\left\langle y_{t}\right\rangle_{t=1}^{\infty}$ of $\left\langle u_{t}\right\rangle_{t=1}^{\infty}$ such that for each $t \in \mathbb{N}$, $\min \left(\operatorname{supp}\left(y_{t+1}\right)\right) \geqslant 13+\max \left(\operatorname{supp}\left(y_{t}\right)\right)$. Using [5, Corollary 5.15], choose a sum subsystem $\left\langle z_{t}\right\rangle_{t=1}^{\infty}$ of $\left\langle y_{t}\right\rangle_{t=1}^{\infty}$ such that $\mathrm{FS}\left(\left\langle z_{t}\right\rangle_{t=1}^{\infty}\right)$ is monochrome.

\section{Definition 3.4.}

(a) $V=\left\{\boldsymbol{v} \in\{0,1,2, \ldots, p-1\}^{11}:\left(v_{1}, v_{2}, \ldots, v_{6}\right) \neq \mathbf{0}\right\}$.

(b) If $x \in \mathbb{Z} \backslash\{0\}$, then

$$
\begin{gathered}
G(x)=\left\{(t, u, \boldsymbol{v}) \in \mathbb{N} \times\{1,2, \ldots, p-1\} \times V: u 00 \ldots 0 v_{1} v_{2} v_{3} \cdots v_{11}\right. \\
\text { occurs in the base }-p \text { expansion of } x \text {, with } u \\
\text { in location } t, v_{1} \text { in a location which is a multiple } \\
\text { of } \left.6 \text { and at least one zero occurring between } u \text { and } v_{1}\right\}
\end{gathered}
$$


A gap of $x$ is any member of $G(x)$. We shall refer to $(t, u, \boldsymbol{v}) \in G(x)$ as a $(u, \boldsymbol{v})$-gap of $x$. The following simple lemma is the key to our counting of gaps.

Lemma 3.5. Let $\left|x_{1}\right|>p^{11}$ and assume that

$$
\max \left(\operatorname{supp}\left(x_{1}\right)\right)+11 \leqslant s=\min \left(\operatorname{supp}\left(x_{2}\right)\right),
$$

then $G\left(x_{1}+x_{2}\right)=G\left(x_{1}\right) \cup G\left(x_{2}\right) \cup\left\{\left(s, \rho\left(x_{2}\right), \lambda\left(x_{1}\right)\right)\right\}$.

Proof. We leave most of the details to the reader, only pointing out the two places where we use the assumption that $\max \left(\operatorname{supp}\left(x_{1}\right)\right)+11 \leqslant \min \left(\operatorname{supp}\left(x_{2}\right)\right)$. Let $r=\max \left(\operatorname{supp}\left(x_{1}\right)\right)$. If $(t, u, \boldsymbol{v})$ is a gap of $x_{2}$ so that $u 00 \cdots 0 v_{1} v_{2} v_{3} \cdots v_{11}$ occurs in the base $-p$ expansion of $x_{2}$, with $u$ in location $t$ and $v_{1}$ in location $j$, then $j \geqslant s \geqslant r+11$ so $u 00 \cdots 0 v_{1} v_{2} v_{3} \cdots v_{11}$ occurs in the expansion of $x_{1}+x_{2}$, with $u$ in location $t$.

Similarly, if $(t, u, \boldsymbol{v})$ is a gap of $x_{1}+x_{2}$ with $t>s$, so that $u 00 \cdots 0 v_{1} v_{2} v_{3} \cdots v_{11}$ occurs in the expansion of $x_{1}+x_{2}$, with $u$ in location $t$, and $v_{1}$ occurs in location $j$, then $j \geqslant s \geqslant r+11$, so none of the digits of $\boldsymbol{v}$ come from $x_{1}$ and thus $(t, u, \boldsymbol{v})$ is a gap of $x_{2}$.

Definition 3.6. Let $x \in \mathbb{Z} \backslash\{0\}$.

(a) For $(u, \boldsymbol{v}) \in\{1,2, \ldots, p-1\} \times V, G_{(u, v)}(x)=\{t \in \mathbb{N}:(t, u, \boldsymbol{v}) \in G(x)\}$.

(b) For $(u, \boldsymbol{v}) \in\{1,2, \ldots, p-1\} \times V, g_{(u, v)}(x)=\left|G_{(u, \boldsymbol{v})}(x)\right|$.

(c) $P(x)=\left\{(u, \boldsymbol{v}) \in\{1,2, \ldots, p-1\} \times V: \pi\left(g_{(u, v)}(x)\right) \in\left\{1,2, \ldots, \frac{1}{2} k\right\}\right\}$.

Thus $G_{(u, v)}(x)$ is the set of locations of $(u, \boldsymbol{v})$-gaps of $x$ and $g_{(u, v)}(x)$ is the number of $(u, \boldsymbol{v})$-gaps of $x$. We shall only be concerned with $(u, \boldsymbol{v})$-gaps of $x$ for $(u, \boldsymbol{v}) \in P(x)$. We pause to give an informal description of the procedure we shall follow to prove that (a) implies (b) in Theorem 3.1.

Let $x=a_{n} w_{n}+\cdots+a_{2} w_{2}+a_{1} w_{1}$, where $\left\langle w_{t}\right\rangle_{t=1}^{\infty}$ is a suitable sum subsystem of $\left\langle x_{t}\right\rangle_{t=1}^{\infty}$. We count gaps in the expansion of $x$. What is a bit confusing is that we have to do this more than once.

(1) Firstly, for a given $(u, \boldsymbol{v})$, we count the number of corresponding gaps in order to decide whether $(u, \boldsymbol{v})$ is in $P(x)$ (i.e. whether the number of $(u, \boldsymbol{v})$-gaps is less than or equal to $\left.\frac{1}{2} k(\bmod k)\right)$.

(2) Then, for each gap $(t, u, \boldsymbol{v}) \in G(x)$, with $(u, \boldsymbol{v})$ in $P(x)$, we count the number of gaps in $P(x)$ which occur to the right of the given one.

(3) Then, keeping $(u, \boldsymbol{v})$ fixed, for each $i \in\{0,1, \ldots, n-2\}$, we count the number of values of $t$ for which the number obtained in $(2)$ is equal to $i(\bmod k)$.

(4) Finally, we ask whether the number obtained in $(3)$ is equal to $1(\bmod k)$. If it is, the gap which occurs between $a_{i+2} w_{i+2}$ and $a_{i+1} w_{i+1}$ is a $(u, \boldsymbol{v})$-gap. 
To indicate why this works, consider the following.

Firstly, $(u, v)$ is in $P(x)$ if and only if it occurs between $a_{i+1} w_{i+1}$ and $a_{i} w_{i}$ for some $i$. If we look at the expansion of $x$ and make the simple-minded assumption that the gaps in $P(x)$ occur only in this way, and never occur internally inside the expansion of some $a_{i} w_{i}$, then the gap between $a_{i+2} w_{i+2}$ and $a_{i+1} w_{i+1}$ is distinguished from the others because it is the only one in $P(x)$ with $i$ gaps of $P(x)$ to its right. Of course, this assumption is likely to be false. However, we get the same answer in (4) as we would if it were true. The reason is that, for the gap between $a_{i+1} w_{i+1}$ and $a_{i} w_{i}$, the number of internal gaps in $P(x)$ to its right is congruent to $0(\bmod k)$. So, whether this gap is counted in $(3)$ or not is unaffected by the internal gaps. Furthermore, the number of internal gaps counted in $(3)$ is congruent to $0(\bmod k)$. So the answer in $(4)$ is unaffected by the internal gaps.

Lemma 3.7. Let $\left\langle x_{t}\right\rangle_{t=1}^{\infty}$ be a sequence in $\mathbb{Z} \backslash\{0\}$ such that

$$
\left|x_{1}\right|>p^{11} \text { and } \max \left(\operatorname{supp}\left(x_{t}\right)\right)+11 \leqslant \min \left(\operatorname{supp}\left(x_{t+1}\right)\right) \quad \text { for every } t \in \mathbb{N} .
$$

Suppose that there exist $u \in\{1,2, \ldots, p-1\}$ and $\boldsymbol{v} \in V$ such that $\rho(x)=u$ and $\lambda(x)=\boldsymbol{v}$ for every $x \in \operatorname{FS}\left(\left\langle x_{t}\right\rangle_{t=1}^{\infty}\right)$. Let $w \in\{1,2, \ldots, p-1\}$, let $\boldsymbol{z} \in V$, let $r \in\{0,1, \ldots, k-1\}$, and assume that $g_{(w, \boldsymbol{z})}(x) \equiv r(\bmod k)$ for each $x \in \operatorname{FS}\left(\left\langle x_{t}\right\rangle_{t=1}^{\infty}\right)$.

(a) If $(w, \boldsymbol{z}) \neq(u, \boldsymbol{v})$, then $r=0$.

(b) If $(w, \boldsymbol{z})=(u, \boldsymbol{v})$, then $r=k-1$.

Proof. If $(w, \boldsymbol{z}) \neq(u, \boldsymbol{v})$, then $G_{(w, \boldsymbol{z})}\left(x_{1}+x_{2}\right)=G_{(w, \boldsymbol{z})}\left(x_{1}\right) \cup G_{(w, \boldsymbol{z})}\left(x_{2}\right)$ and so $g_{(w, \boldsymbol{z})}\left(x_{1}+x_{2}\right)=g_{(w, \boldsymbol{z})}\left(x_{1}\right)+g_{(w, \boldsymbol{z})}\left(x_{2}\right)$. If $(w, \boldsymbol{z})=(u, \boldsymbol{v})$ and $\min \left(\operatorname{supp}\left(x_{2}\right)\right)=s$, then $G_{(w, \boldsymbol{z})}\left(x_{1}+x_{2}\right)=G_{(w, \boldsymbol{z})}\left(x_{1}\right) \cup G_{(w, \boldsymbol{z})}\left(x_{2}\right) \cup\{s\}$ and so $g_{(w, \boldsymbol{z})}\left(x_{1}+x_{2}\right)=g_{(w, \boldsymbol{z})}\left(x_{1}\right)+$ $g_{(w, \boldsymbol{z})}\left(x_{2}\right)+1$.

Lemma 3.8. Let $\left\langle x_{t}\right\rangle_{t=1}^{\infty}$ be a sequence in $\mathbb{N}$ such that $x_{1}>p^{11}$ and $\max \left(\operatorname{supp}\left(x_{t}\right)\right)+$ $13 \leqslant \min \left(\operatorname{supp}\left(x_{t+1}\right)\right)$ for each $t \in \mathbb{N}$. Suppose that $\rho\left(a_{i} x\right)=\rho\left(a_{i} x^{\prime}\right)$ and $\lambda\left(a_{i} x\right)=\lambda\left(a_{i} x^{\prime}\right)$ for all $x, x^{\prime} \in \mathrm{FS}\left(\left\langle x_{t}\right\rangle_{t=1}^{\infty}\right)$ and all $i \in\{1,2, \ldots, n\}$. Suppose also that $g_{(w, \boldsymbol{z})}\left(a_{i} x\right) \equiv$ $g_{(w, \boldsymbol{z})}\left(a_{i} x^{\prime}\right)(\bmod k)$ for all $x, x^{\prime} \in \operatorname{FS}\left(\left\langle x_{t}\right\rangle_{t=1}^{\infty}\right)$, all $i \in\{1,2, \ldots, n\}$, and all $(w, \boldsymbol{z}) \in$ $\{1,2, \ldots, p-1\} \times V$. If $x \in \mathrm{FS}\left(\left\langle x_{t}\right\rangle_{t=1}^{\infty}\right), j \in\{1,2, \ldots, n-1\}, w=\rho\left(a_{j+1} x\right)$, and $\boldsymbol{z}=\lambda\left(a_{j} x\right)$, then $g_{(w, \boldsymbol{z})}\left(a_{i} x\right) \equiv 0(\bmod k)$ for all $i \in\{1,2, \ldots, n\}$.

Proof. Let $i \in\{1,2, \ldots, n\}$ and notice that the sequence $\left\langle a_{i} x_{t}\right\rangle_{t=1}^{\infty}$ satisfies the hypotheses of Lemma 3.7. (Given $t \in \mathbb{N}$, by Corollary 2.3 we have that $\max \left(\operatorname{supp}\left(a_{i} x_{t}\right)\right)+$ $11 \leqslant \max \left(\operatorname{supp}\left(x_{t}\right)\right)+13 \leqslant \min \left(\operatorname{supp}\left(x_{t+1}\right)\right)=\min \left(\operatorname{supp}\left(a_{i} x_{t+1}\right)\right)$.

Let $j \in\{1,2, \ldots, n-1\}$, let $w=\rho\left(a_{j+1} x_{1}\right)$, and let $\boldsymbol{z}=\lambda\left(a_{j} x_{1}\right)$. By Lemma 3.7 it suffices to show that $(w, \boldsymbol{z}) \neq\left(\rho\left(a_{i} x_{1}\right), \lambda\left(a_{i} x_{1}\right)\right)$. Suppose instead that $\rho\left(a_{j+1} x_{1}\right)=$ $\rho\left(a_{i} x_{1}\right)$ and $\lambda\left(a_{j} x_{1}\right)=\lambda\left(a_{i} x_{1}\right)$. Then by Lemma 2.5 (i) we have immediately that $a_{j+1}=$ $a_{i}$. By Lemma 2.5 (ii), with $x=y=x_{1}$ and $c=1$, we have that $a_{j}=a_{i}$. This contradicts the fact that $\boldsymbol{a}$ is a compressed sequence. 
We are now in a position to complete the proof of Theorem 3.1 by showing that (a) implies (b).

Proof that (a) implies (b). Recall that we have been assuming that we have compressed sequences $\boldsymbol{a}=\left\langle a_{1}, a_{2}, \ldots, a_{n}\right\rangle$ and $\boldsymbol{b}=\left\langle b_{1}, b_{2}, \ldots, b_{m}\right\rangle$ with entries from $\mathbb{Z} \backslash\{0\}$ such that whenever $\mathbb{Z}$ is finitely coloured there exist sequences $\left\langle x_{t}\right\rangle_{t=1}^{\infty}$ and $\left\langle y_{t}\right\rangle_{t=1}^{\infty}$ in $\mathbb{N}$ with $\operatorname{MT}\left(\boldsymbol{a},\left\langle x_{t}\right\rangle_{t=1}^{\infty}\right) \cup \operatorname{MT}\left(\boldsymbol{b},\left\langle y_{t}\right\rangle_{t=1}^{\infty}\right)$ monochrome. We show first that we may assume that $a_{n}=b_{m} \in \mathbb{N}$ (and then show that $\boldsymbol{a}=\boldsymbol{b}$ ). To see this note that $a_{n}$ and $b_{m}$ have the same sign. (If $\left\langle x_{t}\right\rangle_{t=1}^{\infty}$ is a sequence in $\mathbb{N}$ and $F \in \mathcal{P}_{f}(\mathbb{N})$ such that $\min F \geqslant n$ and $\sum_{t \in F} x_{t}>\left|\sum_{i=1}^{n-1} a_{i} x_{i}\right|$, then $a_{n} \sum_{t \in F} x_{t}+\sum_{i=1}^{n-1} a_{i} x_{i}$ has the same sign as $a_{n}$.) Also, if $\mathbb{Z}=\bigcup_{i=1}^{r} B_{i}$, then $\mathbb{Z}=\bigcup_{i=1}^{r}\left(-B_{i}\right)$, so statement (a) holds for $\boldsymbol{a}$ and $\boldsymbol{b}$ if and only if it holds for $-\boldsymbol{a}$ and $\boldsymbol{- b}$. Thus we may assume that $a_{n}$ and $b_{m}$ are positive.

Let $\boldsymbol{c}=b_{m} \boldsymbol{a}$ and let $\boldsymbol{d}=a_{n} \boldsymbol{b}$. We claim that $\boldsymbol{c}$ and $\boldsymbol{d}$ satisfy statement (a). To see this, let $r \in \mathbb{N}$ and let $\mathbb{Z}=\bigcup_{i=1}^{r} B_{i}$. Pick $i \in\{1,2, \ldots, r\}$ and sequences $\left\langle x_{t}\right\rangle_{t=1}^{\infty}$ and $\left\langle y_{t}\right\rangle_{t=1}^{\infty}$ in $\mathbb{N}$ with $\operatorname{MT}\left(\boldsymbol{a},\left\langle x_{t}\right\rangle_{t=1}^{\infty}\right) \cup \operatorname{MT}\left(\boldsymbol{b},\left\langle y_{t}\right\rangle_{t=1}^{\infty}\right) \subseteq B_{i}$. By passing to sum subsystems we may presume (using Lemma 3.3) that $\operatorname{FS}\left(\left\langle x_{t}\right\rangle_{t=1}^{\infty}\right) \subseteq b_{m} \mathbb{N}$ and $\operatorname{FS}\left(\left\langle y_{t}\right\rangle_{t=1}^{\infty}\right) \subseteq a_{n} \mathbb{N}$. For $t \in \mathbb{N}$, let $u_{t}=\left(x_{t} / b_{m}\right)$ and $v_{t}=\left(y_{t} / a_{n}\right)$. Then $\operatorname{MT}\left(\boldsymbol{c},\left\langle u_{t}\right\rangle_{t=1}^{\infty}\right)=\operatorname{MT}\left(\boldsymbol{a},\left\langle x_{t}\right\rangle_{t=1}^{\infty}\right)$ and $\operatorname{MT}\left(\boldsymbol{d},\left\langle v_{t}\right\rangle_{t=1}^{\infty}\right)=\operatorname{MT}\left(\boldsymbol{b},\left\langle y_{t}\right\rangle_{t=1}^{\infty}\right)$. Therefore, we may assume that $a_{n}=b_{m} \in \mathbb{N}$ as claimed.

Now for $x \in \mathbb{Z} \backslash\{0\}$, let $G_{P}(x)=\{(t, u, \boldsymbol{v}) \in G(x):(u, \boldsymbol{v}) \in P(x)\}$. For $x \in \mathbb{Z} \backslash\{0\}$ and $t \in \mathbb{N}$, let $R_{t}(x)=\left\{\left(t^{\prime}, u^{\prime}, \boldsymbol{v}^{\prime}\right) \in G_{P}(x): t^{\prime}<t\right\}$. For $x \in \mathbb{Z} \backslash\{0\}$ and $i \in\{0,1, \ldots, k-1\}$, let

$$
S_{i}(x)=\left\{(t, u, \boldsymbol{v}) \in G_{P}(x): \pi\left(\left|R_{t}(x)\right|\right)=i\right\}
$$

and

$$
T_{i}(x)=\left\{(u, \boldsymbol{v}) \in\{1,2, \ldots, p-1\} \times V: \pi\left(\left|\left\{t \in \mathbb{N}:(t, u, \boldsymbol{v}) \in S_{i}(x)\right\}\right|\right)=1\right\}
$$

We define a colouring $\varphi$ of $\mathbb{Z}$ as follows. For $x, y \in \mathbb{Z}, \varphi(x)=\varphi(y)$ if and only if either $x=y=0$ or $\lambda(x)=\lambda(y), \rho(x)=\rho(y), \pi\left(g_{(u, v)}(x)\right)=\pi\left(g_{(u, v)}(y)\right)$ for every $(u, \boldsymbol{v}) \in\{1,2, \ldots, p-1\} \times V$, and $T_{i}(x)=T_{i}(y)$ for every $i \in\{0,1, \ldots, k-1\}$. Notice that $\varphi$ is a finite colouring of $\mathbb{Z}$. Pick sequences $\left\langle x_{t}\right\rangle_{t=1}^{\infty}$ and $\left\langle y_{t}\right\rangle_{t=1}^{\infty}$ in $\mathbb{N}$ such that $\varphi(x)=\varphi(y)$ for every $x \in \operatorname{MT}\left(\boldsymbol{a},\left\langle x_{t}\right\rangle_{t=1}^{\infty}\right)$ and every $y \in \operatorname{MT}\left(\boldsymbol{b},\left\langle y_{t}\right\rangle_{t=1}^{\infty}\right)$.

Now define a colouring $\psi$ of $\mathbb{Z}$ as follows. For $x, y \in \mathbb{Z}, \psi(x)=\psi(y)$ if and only if either $x=y=0$ or

(1) $\rho(x)=\rho(y)$ and $\lambda(x)=\lambda(y)$;

(2) for all $i \in\{1,2, \ldots, n\}, \rho\left(a_{i} x\right)=\rho\left(a_{i} y\right)$ and $\lambda\left(a_{i} x\right)=\lambda\left(a_{i} y\right)$;

(3) for all $i \in\{1,2, \ldots, m\}, \rho\left(b_{i} x\right)=\rho\left(b_{i} y\right)$ and $\lambda\left(b_{i} x\right)=\lambda\left(b_{i} y\right)$; 
(4) for all $(u, \boldsymbol{v}) \in\{1,2, \ldots, p-1\} \times V$ and all $i \in\{1,2, \ldots, n\}, g_{(u, v)}\left(a_{i} x\right) \equiv$ $g_{(u, v)}\left(a_{i} y\right)(\bmod k)$; and

(5) for all $(u, \boldsymbol{v}) \in\{1,2, \ldots, p-1\} \times V$ and all $i \in\{1,2, \ldots, m\}, g_{(u, v)}\left(b_{i} x\right) \equiv$ $g_{(u, \boldsymbol{v})}\left(b_{i} y\right)(\bmod k)$.

Using Lemma 3.3 and passing to sum subsystems, we may presume that

(a) $x_{1}>p^{11}$ and $y_{1}>p^{11}$;

(b) for each $t \in \mathbb{N}, \min \left(\operatorname{supp}\left(x_{t+1}\right)\right) \geqslant 13+\max \left(\operatorname{supp}\left(x_{t}\right)\right)$ and $\min \left(\operatorname{supp}\left(y_{t+1}\right)\right) \geqslant$ $13+\max \left(\operatorname{supp}\left(y_{t}\right)\right) ;$ and

(c) for all $x, x^{\prime} \in \mathrm{FS}\left(\left\langle x_{t}\right\rangle_{t=1}^{\infty}\right)$ and all $y, y^{\prime} \in \mathrm{FS}\left(\left\langle y_{t}\right\rangle_{t=1}^{\infty}\right)$, one has $\psi(x)=\psi\left(x^{\prime}\right)$ and $\psi(y)=\psi\left(y^{\prime}\right)$.

We have some $P \subseteq\{1,2, \ldots, p-1\} \times V$ such that for all $x \in \operatorname{MT}\left(\boldsymbol{a},\left\langle x_{t}\right\rangle_{t=1}^{\infty}\right)$ and all $y \in \operatorname{MT}\left(\boldsymbol{b},\left\langle y_{t}\right\rangle_{t=1}^{\infty}\right), P(x)=P(y)=P$, because $\pi\left(g_{(u, v)}(x)\right)=\pi\left(g_{(u, v)}(y)\right)$ for all $(u, \boldsymbol{v}) \in\{1,2, \ldots, p-1\} \times V$. Let $Q=\left\{\left(\rho\left(a_{j+1} x_{j+1}\right), \lambda\left(a_{j} x_{j}\right)\right): j \in\{1,2, \ldots, n-1\}\right\}$. We claim that $P=Q$. To see this, note that by Lemma 3.7 and conditions (2) and (4) of the definition of $\psi, \pi\left(g_{(u, v)}\left(a_{i} x_{i}\right)\right) \in\{0, k-1\}$ for all $(u, v) \in\{1,2, \ldots, p-1\} \times V$ and all $i \in\{1,2, \ldots, n\}$. By Lemma 3.8, if $(u, v) \in Q$, then $\pi\left(g_{(u, v)}\left(a_{i} x_{i}\right)\right)=0$ for all $i \in\{1,2, \ldots, n\}$.

Now let $x=a_{n} x_{n}+a_{n-1} x_{n-1}+\cdots+a_{1} x_{1}$, so that $P(x)=P$. For any $(u, \boldsymbol{v}) \in$ $\{1,2, \ldots, p-1\} \times V$, we have

$g_{(u, \boldsymbol{v})}(x)=\sum_{i=1}^{n} g_{(u, \boldsymbol{v})}\left(a_{i} x_{i}\right)+\left|\left\{j \in\{1,2, \ldots, n-1\}:(u, \boldsymbol{v})=\left(\rho\left(a_{j+1} x_{j+1}\right), \lambda\left(a_{j}, x_{j}\right)\right)\right\}\right|$.

Thus

$$
\begin{aligned}
& \text { if }(u, \boldsymbol{v}) \in Q, \text { then } \pi\left(g_{(u, v)}(x)\right)=\mid\{j \in\{1,2, \ldots, n-1\}: \\
& \left.\qquad(u, v)=\left(\rho\left(a_{j+1} x_{j+1}\right), \lambda\left(a_{j}, x_{j}\right)\right)\right\} \mid .
\end{aligned}
$$

On the other hand, if $(u, \boldsymbol{v}) \notin Q$, then $g_{(u, v)}(x)=\sum_{i=1}^{n} g_{(u, v)}\left(a_{i} x_{i}\right)$, so either

$$
\pi\left(g_{(u, v)}(x)\right)=0 \quad \text { or } \quad \pi\left(g_{(u, v)}(x)\right) \in\{k-n, k-n+1, \ldots, k-1\}
$$

so that $(u, v) \notin P$. Thus $P=Q$ as claimed. Similarly, $P=\left\{\left(\rho\left(b_{j+1} y_{j+1}\right), \lambda\left(b_{j} y_{j}\right)\right): j \in\right.$ $\{1,2, \ldots, m-1\}\}$.

Now using $(*)$ and the corresponding assertion for $y=b_{1} y_{1}+b_{2} y_{2}+\cdots+b_{m} y_{m}$, we have

$$
n-1=\sum_{(u, \boldsymbol{v}) \in P(x)} \pi\left(g_{(u, \boldsymbol{v})}(x)\right)=\sum_{(u, \boldsymbol{v}) \in P(y)} \pi\left(g_{(u, \boldsymbol{v})}(y)\right)=m-1,
$$

so $n=m$. 
For $t \in \mathbb{N}$ and $z \in \mathbb{Z} \backslash\{0\}$, let

$$
\delta_{t}(z)=\left\{\left(t^{\prime}, u^{\prime}, \boldsymbol{v}^{\prime}\right) \in G(z): t^{\prime}<t \quad \text { and } \quad\left(u^{\prime}, \boldsymbol{v}^{\prime}\right) \in Q\right\} .
$$

Given $(u, \boldsymbol{v}) \in Q, i \in\{0,1, \ldots, k-1\}$ and $z \in \mathbb{Z} \backslash\{0\}$, let

$$
\gamma_{(i, u, \boldsymbol{v})}(z)=\left\{t \in \mathbb{N}:(t, u, \boldsymbol{v}) \in G(z) \text { and } \pi\left(\left|\delta_{t}(z)\right|\right)=i\right\} .
$$

Using Lemma 3.3, choose a sum subsystem $\left\langle w_{t}\right\rangle_{t=1}^{\infty}$ of $\left\langle x_{t}\right\rangle_{t=1}^{\infty}$ such that for all $w, w^{\prime} \in \mathrm{FS}\left(\left\langle w_{t}\right\rangle_{t=1}^{\infty}\right)$, all $(u, \boldsymbol{v}) \in Q$, all $s \in\{1,2, \ldots, n\}$ and all $i \in\{0,1, \ldots, k-1\}$, $\left|\gamma_{(i, u, \boldsymbol{v})}\left(a_{s} w\right)\right| \equiv\left|\gamma_{(i, u, v)}\left(a_{s} w^{\prime}\right)\right|(\bmod k)$.

Let $(u, v) \in Q$, let $s \in\{1,2, \ldots, n\}$, and let $i \in\{0,1, \ldots, k-1\}$. We claim that $\left|\gamma_{(i, u, v)}\left(a_{s} w\right)\right| \equiv 0(\bmod k)$ for all $w \in \mathrm{FS}\left(\left\langle w_{t}\right\rangle_{t=1}^{\infty}\right)$. For this it suffices to show that $\gamma_{(i, u, \boldsymbol{v})}\left(a_{s} w_{2}+a_{s} w_{1}\right)=\gamma_{(i, u, \boldsymbol{v})}\left(a_{s} w_{2}\right) \cup \gamma_{(i, u, \boldsymbol{v})}\left(a_{s} w_{1}\right)$.

We note that $\left(\rho\left(a_{s} w_{2}\right), \lambda\left(a_{s} w_{1}\right)\right) \notin Q$. To see this, suppose instead that

$$
\left(\rho\left(a_{s} w_{2}\right), \lambda\left(a_{s} w_{1}\right)\right)=\left(\rho\left(a_{j+1} x_{j+1}\right), \lambda\left(a_{j} x_{j}\right)\right) \text { for some } j \in\{1,2, \ldots, n-1\} .
$$

Since $w_{1}, w_{2} \in \operatorname{FS}\left(\left\langle x_{t}\right\rangle_{t=1}^{\infty}\right)$, we have that $\rho\left(a_{j+1} x_{j+1}\right)=\rho\left(a_{s} w_{2}\right)=\rho\left(a_{s} x_{j+1}\right)$ and $\lambda\left(a_{j} x_{j}\right)=\lambda\left(a_{s} w_{1}\right)=\lambda\left(a_{s} x_{j}\right)$. But then by Lemma 2.5, $a_{j+1}=a_{s}=a_{j}$, contradicting the fact that $\boldsymbol{a}$ is a compressed sequence. Thus since $\left(\rho\left(a_{s} w_{2}\right), \lambda\left(a_{s} w_{1}\right)\right) \notin Q$,

$$
\begin{aligned}
\gamma_{(i, u, \boldsymbol{v})}\left(a_{s} w_{2}+a_{s} w_{1}\right)=\left\{t \in \mathbb{N}:(t, u, \boldsymbol{v}) \in G\left(a_{s} w_{2}\right) \text { and } \pi\left(\left|\delta_{t}\left(a_{s} w_{2}+a_{s} w_{1}\right)\right|\right)=i\right\} \\
\cup\left\{t \in \mathbb{N}:(t, u, \boldsymbol{v}) \in G\left(a_{s} w_{1}\right) \text { and } \pi\left(\left|\delta_{t}\left(a_{s} w_{2}+a_{s} w_{1}\right)\right|\right)=i\right\}
\end{aligned}
$$

Now, if $(t, u, \boldsymbol{v}) \in G\left(a_{s} w_{1}\right)$, then $\delta_{t}\left(a_{s} w_{2}+a_{s} w_{1}\right)=\delta_{t}\left(a_{s} w_{1}\right)$. If $(t, u, \boldsymbol{v}) \in G\left(a_{s} w_{2}\right)$, then (again using the fact that $\left.\left(\rho\left(a_{s} w_{2}\right), \lambda\left(a_{s} w_{1}\right)\right) \notin Q\right)$ we have $\delta_{t}\left(a_{s} w_{2}+a_{s} w_{1}\right)=$ $\delta_{t}\left(a_{s} w_{2}\right) \cup \delta_{t}\left(a_{s} w_{1}\right)$. Also, for $(t, u, \boldsymbol{v}) \in G\left(a_{s} w_{2}\right)$,

$$
\delta_{t}\left(a_{s} w_{1}\right)=\bigcup_{\left(u^{\prime}, \boldsymbol{v}^{\prime}\right) \in Q}\left\{\left(t^{\prime}, u^{\prime}, \boldsymbol{v}^{\prime}\right): t^{\prime} \in G_{\left(u^{\prime}, \boldsymbol{v}^{\prime}\right)}\left(a_{s} w_{1}\right)\right\}
$$

and so $\left|\delta_{t}\left(a_{s} w_{1}\right)\right|=\sum_{\left(u^{\prime}, \boldsymbol{v}^{\prime}\right) \in Q} g_{\left(u, \boldsymbol{v}^{\prime}\right)}\left(a_{s} w_{1}\right) \equiv 0(\bmod k)$. Thus if $(t, u, \boldsymbol{v}) \in G\left(a_{s} w_{2}\right)$, we have $\pi\left(\left|\delta_{t}\left(a_{s} w_{2}+a_{s} w_{1}\right)\right|\right)=\pi\left(\left|\delta_{t}\left(a_{s} w_{2}\right)\right|\right)$. Therefore, $\gamma_{(i, u, v)}\left(a_{s} w_{2}+a_{s} w_{1}\right)=$ $\gamma_{(i, u, \boldsymbol{v})}\left(a_{s} w_{2}\right) \cup \gamma_{(i, u, \boldsymbol{v})}\left(a_{s} w_{1}\right)$ as required.

We shall complete the proof by showing that for any $x \in \operatorname{MT}\left(\boldsymbol{a},\left\langle x_{t}\right\rangle_{t=1}^{\infty}\right)$, any $z \in$ $\operatorname{FS}\left(\left\langle x_{t}\right\rangle_{t=1}^{\infty}\right)$ and any $i \in\{0,1, \ldots, n-2\}$,

$$
T_{i}(x)=\left\{\left(\rho\left(a_{i+2} z\right), \lambda\left(a_{i+1} z\right)\right)\right\} .
$$

Assume for now that we have done this. It will then follow similarly that for any $y \in \operatorname{MT}\left(\boldsymbol{a},\left\langle y_{t}\right\rangle_{t=1}^{\infty}\right)$, any $q \in \operatorname{FS}\left(\left\langle y_{t}\right\rangle_{t=1}^{\infty}\right)$ and any $i \in\{0,1, \ldots, n-2\}, T_{i}(y)=$ 
$\left\{\left(\rho\left(b_{i+2} q\right), \lambda\left(b_{i+1} q\right)\right)\right\}$. Since for such $x, y$ and $i$, we have $T_{i}(x)=T_{i}(y)$, we must then have in particular that $\lambda\left(a_{i+1} x_{n}\right)=\lambda\left(b_{i+1} y_{n}\right)$. We also have that

$$
\begin{aligned}
\lambda\left(a_{n} x_{n}\right) & =\lambda\left(a_{n} x_{n}+a_{n-1} x_{n-1}+\cdots+a_{1} x_{1}\right) \\
& =\lambda\left(b_{n} y_{n}+b_{n-1} y_{n-1}+\cdots+b_{1} y_{1}\right)=\lambda\left(b_{n} y_{n}\right)=\lambda\left(a_{n} y_{n}\right) .
\end{aligned}
$$

Thus by Lemma 2.5 (ii), we will have that $a_{i+1}=b_{i+1}$ for each $i \in\{0,1, \ldots, n-2\}$. Since we already know that $a_{n}=b_{n}$, we will then have $\boldsymbol{a}=\boldsymbol{b}$.

To establish $(\dagger)$, let $x=a_{n} w_{n}+a_{n-1} w_{n-1}+\cdots+a_{1} w_{1}$. We show that $T_{i}(x)=$ $\left\{\left(\rho\left(a_{i+2} w_{i+2}\right), \lambda\left(a_{i+1} w_{i+1}\right)\right)\right\}$ for each $i \in\{0,1, \ldots, n-2\}$. Notice that if $i \in\{0,1, \ldots, n-$ $2\}$ and $(u, \boldsymbol{v}) \in T_{i}(x)$, then $\left\{t \in \mathbb{N}:(t, u, \boldsymbol{v}) \in S_{i}(x)\right\} \neq \emptyset$ and so $(u, \boldsymbol{v}) \in P(x)=Q$. Consequently, for each $i \in\{0,1, \ldots, n-2\}$,

$$
T_{i}(x)=\left\{(u, \boldsymbol{v}) \in Q: \pi\left(\left|\left\{t \in \mathbb{N}:(t, u, \boldsymbol{v}) \in S_{i}(x)\right\}\right|\right)=1\right\} .
$$

Let $(u, \boldsymbol{v})=\left(\rho\left(a_{i+2} w_{i+2}\right), \lambda\left(a_{i+1} w_{i+1}\right)\right)$, where $i \in\{0,1, \ldots, n-2\}$. We consider $\{t \in$ $\left.\mathbb{N}:(t, u, \boldsymbol{v}) \in S_{i}(x)\right\}$. If $t=\min \left(\operatorname{supp}\left(a_{i+2} w_{i+2}\right)\right)$, then $(t, u, \boldsymbol{v}) \in S_{i}(x)$, because it follows from Lemma 3.8 that $g_{(u, v)}\left(a_{s} w_{s}\right) \equiv 0(\bmod k)$ for every $s \in\{1,2, \ldots, n\}$. If $(t, u, v) \in$ $G(x)$ and $t=\min \left(\operatorname{supp}\left(a_{j+2} w_{j+2}\right)\right)$, with $j \in\{0,1, \ldots, n-2\} \backslash\{i\}$, then $(t, u, \boldsymbol{v}) \in S_{j}(x)$ and thus $(t, u, \boldsymbol{v}) \notin S_{i}(x)$. If $(t, u, \boldsymbol{v}) \in G\left(a_{s} w_{s}\right)$ for some $s \in\{1,2, \ldots, n\}$, then $(t, u, \boldsymbol{v}) \in$ $S_{i}(x)$ if and only if $t \in \gamma_{(j, u, v)}\left(a_{s} w_{s}\right)$, where $j+s-1 \equiv i(\bmod k)$. We have seen that $\left|\gamma_{(j, u, v)}\left(a_{s} w_{s}\right)\right| \equiv 0(\bmod k)$. So $\left|\left\{t \in \mathbb{N}:(t, u, \boldsymbol{v}) \in S_{i}(x)\right\}\right| \in 1+k \omega$, i.e. $(u, \boldsymbol{v}) \in T_{i}(x)$.

Now let $(w, \boldsymbol{z}) \in P(x) \backslash\{(u, \boldsymbol{v})\}$. Then $(t, w, \boldsymbol{z}) \in S_{i}(x)$ if and only if $t \in \gamma_{(j, w, \boldsymbol{z})}\left(a_{s} w_{s}\right)$ for some $s \in\{1,2, \ldots, n\}$, where $j+s-1 \equiv i(\bmod k)$. Since $\left|\gamma_{(j, w, \boldsymbol{z})}\left(a_{s} w_{s}\right)\right| \equiv 0(\bmod k)$, $\left|\left\{t \in \mathbb{N}:(t, w, \boldsymbol{z}) \in S_{i}(x)\right\}\right| \in k \omega$ and so $(w, \boldsymbol{z}) \notin T_{i}(x)$.

Thus $T_{i}(x)=\{(u, \boldsymbol{v})\}$, and we have established that (†) holds.

In the proof of Theorem 3.1 we used a large number of colours. We observe now that in fact two colours suffice.

Corollary 3.9. Let $\boldsymbol{a}$ and $\boldsymbol{b}$ be finite compressed sequences with entries from $\mathbb{Z} \backslash\{0\}$ and assume that there is no positive rational number $\alpha$ such that $\boldsymbol{b}=\alpha \cdot \boldsymbol{a}$. Then there exist sets $A$ and $B$ such that $\mathbb{Z}=A \cup B$ and there is no sequence $\left\langle x_{i}\right\rangle_{i=1}^{\infty}$ with $\operatorname{MT}\left(\boldsymbol{a},\left\langle x_{i}\right\rangle_{i=1}^{\infty}\right) \subseteq B$ and there is no sequence $\left\langle y_{i}\right\rangle_{i=1}^{\infty}$ with $\operatorname{MT}\left(\boldsymbol{b},\left\langle y_{i}\right\rangle_{i=1}^{\infty}\right) \subseteq A$.

Proof. By Theorem 3.1, pick an $r \in \mathbb{N}$ and sets $\left\langle C_{j}\right\rangle_{j=1}^{r}$ such that $\mathbb{Z}=\bigcup_{j=1}^{r} C_{j}$ and for no $j \in\{1,2, \ldots, r\}$ do there exist sequences $\left\langle x_{i}\right\rangle_{i=1}^{\infty}$ and $\left\langle y_{i}\right\rangle_{i=1}^{\infty}$ with $\operatorname{MT}\left(\boldsymbol{a},\left\langle x_{i}\right\rangle_{i=1}^{\infty}\right) \cup$ $\operatorname{MT}\left(\boldsymbol{b},\left\langle y_{i}\right\rangle_{i=1}^{\infty}\right) \subseteq C_{j}$. Let $A=\bigcup\left\{C_{j}\right.$ : there exists $\left\langle x_{i}\right\rangle_{i=1}^{\infty}$ with $\left.\operatorname{MT}\left(\boldsymbol{a},\left\langle x_{i}\right\rangle_{i=1}^{\infty}\right) \subseteq C_{j}\right\}$ and let $B=\mathbb{N} \backslash A$. By Theorem 1.8 the sets $A$ and $B$ are as required.

\section{Equations in $\beta \mathbb{Z}$}

The results of this paper are intimately related to the algebra in the Stone-Cech compactification $\beta \mathbb{Z}$ of $\mathbb{Z}$. Given any discrete semigroup $(S, \cdot)$, the operation extends to $\beta S$, 
making $(\beta S, \cdot)$ a compact right-topological semigroup with $S$ contained in the topological centre of $\beta S$. We take the points of $\beta S$ to be the ultrafilters on $S$. See [5] for an elementary introduction to this structure, and for the meaning of any unfamiliar terms used here.

In particular, the operations + and $\cdot$ on $\mathbb{Z}$ both extend to $\beta \mathbb{Z}$ making $(\beta \mathbb{Z},+)$ and $(\beta \mathbb{Z}, \cdot)$ right-topological semigroups. The following theorem easily implies our Theorem 1.8 . In this result it is important to note that, for example, $2 \cdot p$ refers to the operation in $(\beta \mathbb{Z}, \cdot)$ and does not mean $p+p$.

Theorem 4.1. Let $\left\langle a_{t}\right\rangle_{t=1}^{n}$ be a sequence in $\mathbb{Z} \backslash\{0\}$, let $p$ be an idempotent in $(\beta \mathbb{N},+)$, and let $q=a_{1} \cdot p+a_{2} \cdot p+\cdots+a_{n} \cdot p$. Let $A \in p$ and $B \in q$. There exists a sequence $\left\langle x_{i}\right\rangle_{i=1}^{\infty}$ in $\mathbb{N}$ with $\operatorname{FS}\left(\left\langle x_{i}\right\rangle_{i=1}^{\infty}\right) \subseteq A$ and $\operatorname{MT}\left(\boldsymbol{a},\left\langle x_{i}\right\rangle_{i=1}^{\infty}\right) \subseteq B$.

Proof. See [7, Lemma 3.4].

To derive Theorem 1.8 from Theorem 4.1, let a sequence $\left\langle y_{i}\right\rangle_{i=1}^{\infty}$ in $\mathbb{N}$ be given, let $r \in \mathbb{N}$ and let $\mathbb{Z}=\bigcup_{j=1}^{r} B_{j}$. By passing to a sum subsystem if necessary, we may presume that for each $i, y_{i+1}>4 \cdot \sum_{t=0}^{i} y_{t}$. By [5, Lemma 5.11], we can pick an idempotent $p$ with $\mathrm{FS}\left(\left\langle y_{i}\right\rangle_{i=1}^{\infty}\right) \in p$ and let $q=a_{1} \cdot p+a_{2} \cdot p+\cdots+a_{n} \cdot p$. Pick $j \in\{1,2, \ldots, r\}$ such that $B_{j} \in q$ and pick a sequence $\left\langle x_{i}\right\rangle_{i=1}^{\infty}$ with $\operatorname{FS}\left(\left\langle x_{i}\right\rangle_{i=1}^{\infty}\right) \subseteq \operatorname{FS}\left(\left\langle y_{i}\right\rangle_{i=1}^{\infty}\right)$ and $\operatorname{MT}\left(\boldsymbol{a},\left\langle y_{i}\right\rangle_{i=1}^{\infty}\right) \subseteq B_{j}$. Since, for each $i$, we had $y_{i+1}>4 \cdot \sum_{t=0}^{i} y_{t}$, one easily sees (using [7, Lemma 3.5], for example) that $\left\langle x_{i}\right\rangle_{i=1}^{\infty}$ is in fact a sum subsystem of $\left\langle y_{i}\right\rangle_{i=1}^{\infty}$.

Maleki observed in Theorem 2.19 in [8] that the results of [3] implied that if $\left\langle a_{1}, a_{2}, \ldots, a_{n}\right\rangle$ and $\left\langle b_{1}, b_{2}, \ldots, b_{m}\right\rangle$ are distinct compressed sequences in $\mathbb{N}$, then the equation $a_{1} \cdot p+a_{2} \cdot p+\cdots+a_{n} \cdot p=b_{1} \cdot p+b_{2} \cdot p+\cdots+b_{m} \cdot p$ has no solutions with $p$ an idempotent in $(\beta \mathbb{N},+)$. (He also showed in [8, Theorem 2.7] that this equation also has no solutions if $p$ is right cancellable in $(\beta \mathbb{N},+)$.) We now see that the corresponding assertion holds where the entries of $\boldsymbol{a}$ and $\boldsymbol{b}$ are allowed to be negative.

Corollary 4.2. Let $\left\langle a_{1}, a_{2}, \ldots, a_{n}\right\rangle$ and $\left\langle b_{1}, b_{2}, \ldots, b_{m}\right\rangle$ be compressed sequences in $\mathbb{Z} \backslash\{0\}$, let $p+p=p \in \beta \mathbb{N}$, and assume that $a_{1} \cdot p+a_{2} \cdot p+\cdots a_{n} \cdot p=b_{1} \cdot p+b_{2} \cdot p+\cdots b_{m} \cdot p$. then $\boldsymbol{a}=\boldsymbol{b}$.

Proof. We show first that it suffices to show that there is some positive rational number $\alpha$ such that $\boldsymbol{b}=\alpha \cdot \boldsymbol{a}$. Let $\alpha=(r / s)$, where $r, s \in \mathbb{N}$. Then, by [5, Lemma 13.1] (which is the only non-trivial instance of the distributive law known to hold in $\beta \mathbb{Z}$ ), we have that

$$
r \cdot\left(a_{1} \cdot p+a_{2} \cdot p+\cdots+a_{n} \cdot p\right)=s \cdot\left(b_{1} \cdot p+b_{2} \cdot p+\cdots+b_{m} \cdot p\right) .
$$

Since also

$$
s \cdot\left(a_{1} \cdot p+a_{2} \cdot p+\cdots+a_{n} \cdot p\right)=s \cdot\left(b_{1} \cdot p+b_{2} \cdot p+\cdots+b_{m} \cdot p\right),
$$

we have by [5, Lemma 6.28] that $r=s$. 
Therefore, by Theorem 3.1, it suffices to show that whenever $r \in \mathbb{N}$ and $\mathbb{Z}=\bigcup_{j=1}^{r} B_{j}$, there exist $j \in\{1,2, \ldots, r\}$ and sequences $\left\langle x_{i}\right\rangle_{i=1}^{\infty}$ and $\left\langle y_{i}\right\rangle_{i=1}^{\infty}$ with $\operatorname{MT}\left(\boldsymbol{a},\left\langle x_{i}\right\rangle_{i=1}^{\infty}\right) \cup$ $\operatorname{MT}\left(\boldsymbol{b},\left\langle y_{i}\right\rangle_{i=1}^{\infty}\right) \subseteq B_{i}$. To this end, pick $j \in\{1,2, \ldots, r\}$ such that $B_{j} \in a_{1} \cdot p+a_{2} \cdot p+$ $\cdots+a_{n} \cdot p$ and apply Theorem 4.1.

We shall see in Theorem 4.4 that one can expand the list of equivalent conditions in Theorem 3.1. One of the added conditions involves idempotents in the smallest ideal $K(\beta \mathbb{N},+)$ of $(\beta \mathbb{N},+)$, the so-called minimal idempotents. These are combinatorially significant because the members of minimal idempotents are central sets and are guaranteed to have rich combinatorial structure (see [5, Chapter 14]).

The following lemma is not new, but does not seem to be in [5].

Lemma 4.3. Let $r$ be an idempotent in $K(\beta \mathbb{N},+)$ and let $k \in \mathbb{N}$. Then $k \cdot r$ is an idempotent in $K(\beta \mathbb{N},+)$.

Proof. The function $p \mapsto k \cdot p$ from $\beta \mathbb{N}$ onto $k \cdot \beta \mathbb{N}$ is a continuous homomorphism. (It is continuous because $\lambda_{k}$ is continuous in $(\beta \mathbb{N}, \cdot)$ and it is a homomorphism by [5, Lemma 13.1].) It maps $K(\beta \mathbb{N},+)$ onto $K(k \cdot \beta \mathbb{N},+)$. Now $k \cdot \beta \mathbb{N}$ contains all the idempotents of $\beta \mathbb{N}$ by [5, Lemma 6.6], and therefore meets $K(\beta \mathbb{N},+)$. It follows from [5, Theorem 1.65] that $K(k \cdot \beta \mathbb{N},+) \subseteq K(\beta \mathbb{N},+)$.

Theorem 4.4. Let $\boldsymbol{a}=\left\langle a_{1}, a_{2}, \ldots, a_{n}\right\rangle$ and $\boldsymbol{b}=\left\langle b_{1}, b_{2}, \ldots, b_{m}\right\rangle$ be finite compressed sequences with entries from $\mathbb{Z} \backslash\{0\}$. The following statements are equivalent.

(a) Whenever $r \in \mathbb{N}$ and $\mathbb{Z}=\bigcup_{i=1}^{r} B_{i}$, there exist $i \in\{1,2, \ldots, r\}$ and sequences $\left\langle x_{t}\right\rangle_{t=1}^{\infty}$ and $\left\langle y_{t}\right\rangle_{t=1}^{\infty}$ in $\mathbb{N}$ with $\operatorname{MT}\left(\boldsymbol{a},\left\langle x_{t}\right\rangle_{t=1}^{\infty}\right) \cup \operatorname{MT}\left(\boldsymbol{b},\left\langle y_{t}\right\rangle_{t=1}^{\infty}\right) \subseteq B_{i}$.

(b) There is a positive rational number $\alpha$ such that $\boldsymbol{b}=\alpha \cdot \boldsymbol{a}$.

(c) There exist idempotents $p$ and $q$ in $K(\beta \mathbb{N},+)$ such that $a_{1} \cdot p+a_{2} \cdot p+\cdots+a_{n} \cdot p=$ $b_{1} \cdot q+b_{2} \cdot q+\cdots+b_{m} \cdot q$.

(d) There exist idempotents $p$ and $q$ in $(\beta \mathbb{N},+)$ such that $a_{1} \cdot p+a_{2} \cdot p+\cdots+a_{n} \cdot p=$ $b_{1} \cdot q+b_{2} \cdot q+\cdots+b_{m} \cdot q$.

Proof. We have by Theorem 3.1 that (a) and (b) are equivalent and that (c) trivially implies (d). By Theorem 4.1, (d) implies (a) (by choosing $i \in\{1,2, \ldots, r\}$ such that $\left.B_{i} \in a_{1} \cdot p+a_{2} \cdot p+\cdots+a_{n} \cdot p=b_{1} \cdot q+b_{2} \cdot q+\cdots+b_{m} \cdot q\right)$.

To see that (b) implies (c), pick $k, l \in \mathbb{N}$ such that $\boldsymbol{b}=(k / l) \cdot \boldsymbol{a}$. Pick any idempotent $r \in K(\beta \mathbb{N})$. Let $p=k \cdot r$ and $q=l \cdot r$. By Lemma 4.3, $p$ and $q$ are idempotents in $K(\beta \mathbb{N},+)$. Then $a_{1} \cdot p+a_{2} \cdot p+\cdots+a_{n} \cdot p=a_{1} \cdot k \cdot r+a_{2} \cdot k \cdot r+\cdots+a_{n} \cdot k \cdot r=$ $b_{1} \cdot l \cdot r+b_{2} \cdot l \cdot r+\cdots+b_{n} \cdot l \cdot r=b_{1} \cdot q+b_{2} \cdot q+\cdots+b_{m} \cdot q$.

We remark that Corollary 3.9 is equivalent to the following statement: if $\boldsymbol{a}$ and $\boldsymbol{b}$ satisfy the hypotheses of this corollary, there exists sets $A$ and $B$ such that $\mathbb{Z}=A \cup B$ and there is no idempotent $p \in \beta \mathbb{N}$ for which $B \in a_{1} \cdot p+a_{2} \cdot p+\cdots+a_{n} \cdot p$ and no idempotent $q \in \beta \mathbb{N}$ for which $A \in b_{1} \cdot q+b_{2} \cdot q+\cdots+b_{m} \cdot q$. This is a property which distinguishes idempotents 
from other elements of $\mathbb{N}^{\star}$. Suppose that $\boldsymbol{a}=\left\langle a_{1}, a_{2}, \ldots, a_{n}\right\rangle$ and $\boldsymbol{b}=\left\langle b_{1}, b_{2}, \ldots, b_{m}\right\rangle$ are arbitrary finite sequences in $\mathbb{Z} \backslash\{0\}$, with $a_{n}, b_{m} \in \mathbb{N}$ and $\sum_{i=1}^{n} a_{i}, \sum_{i=1}^{m} b_{i} \neq 0$. Then it follows from results in [7] that, in any finite colouring of $\mathbb{N}$, there exist $p, q \in \mathbb{N}^{*}$ such that $a_{1} \cdot p+a_{2} \cdot p+\cdots+a_{n} \cdot p$ and $b_{1} \cdot q+b_{2} \cdot q+\cdots+b_{m} \cdot q$ have the same monochrome set as a member. This is even true if we require that $p$ and $q$ have rapidly increasing sets as members, where we call a subset $\left\{t_{n}: n \in \mathbb{N}\right\}$ of $\mathbb{N}$ rapidly increasing if $t_{n+1}-t_{n} \rightarrow \infty$. However, if $p$ and $q$ have rapidly increasing sets as members, it is quite easy to prove that the equation $a_{1} \cdot p+a_{2} \cdot p+\cdots+a_{n} \cdot p=b_{1} \cdot q+b_{2} \cdot q+\cdots+b_{m} \cdot q$ can only hold if $\boldsymbol{b}$ is a positive rational multiple of $\boldsymbol{a}$.

We conclude by modifying [6, Question 1.5] (which remains unanswered) to allow for negative entries.

Question 4.5. Let $\left\langle a_{1}, a_{2}, \ldots, a_{n}\right\rangle$ and $\left\langle b_{1}, b_{2}, \ldots, b_{m}\right\rangle$ be compressed sequences in $\mathbb{Z} \backslash\{0\}$. Suppose that there exists some $p \in \mathbb{N}^{*}$ such that

$$
a_{1} \cdot p+a_{2} \cdot p+\cdots+a_{n} \cdot p=b_{1} \cdot p+b_{2} \cdot p+\cdots+b_{m} \cdot p .
$$

Must it then be true that $\boldsymbol{a}=\boldsymbol{b}$ ?

We note that it can be shown that this equation implies that $a_{1}=b_{1}$ and $a_{n}=b_{m}$. The implication $a_{n}=b_{m}$ was shown in [6] in the case in which $a_{n}, b_{m}>0$, and it is easy to see that we can assume this. The implication $a_{1}=b_{1}$ was also shown in $[\mathbf{6}]$ in the case in which $a_{1}, b_{1}>0$, and the proof in [6] extends easily to the general case.

Acknowledgements. N.H. acknowledges support received from the National Science Foundation (USA) via grant DMS-0070593.

\section{References}

1. B. BordBar AND J. PyM, The set of idempotents in the weakly almost periodic compactification of the integers is not closed, Trans. Am. Math. Soc. 352 (2000), 823-842.

2. W. Deuber, Partitionen und lineare Gleichungssysteme, Math. Z. 133 (1973), 109-123.

3. W. Deuber, N. Hindman, I. Leader and H. Lefmann, Infinite partition regular matrices, Combinatorica 15 (1995), 333-355.

4. N. Hindman AND I. LEADER, Image partition regularity of matrices, Combinatorics Probab. Comput. 2 (1993), 437-463.

5. N. Hindman and D. Strauss, Algebra in the Stone-Čech compactification (Walter de Gruyter, Berlin, 1998).

6. N. Hindman, A. Maleki and D. Strauss, Linear equations in the Stone-Čech compactification of $\mathbb{N}$, Integers $\mathbf{0}$ (2000), \#A02, 1-20.

7. N. Hindman, I. Leader and D. Strauss, Infinite partition regular matrices-solutions in central sets, Trans. Am. Math. Soc., in press.

8. A. MALEKI, Solving equations in $\beta \mathbb{N}$, Semigroup Forum 61 (2000), 373-384.

9. K. Milliken, Ramsey's theorem with sums or unions, J. Combinat. Theory A 18 (1975), 276-290.

10. A. TAYLOR, A canonical partition relation for finite subsets of $\omega, J$. Combinat. Theory A 21 (1976), 137-146. 